\title{
Recurrent pyogenic liver abscess as a presenting manifestation of colorectal cancer
}

\author{
Hee Yeon Kim, Chang Wook Kim, Dong Ryul Kim, Young Wook Cho, Jae-Young Cho, Woo Jung Kim, \\ and Hyung Gyu Choi
}

Division of Gastroenterology and Hepatology, Department of Internal Medicine, College of Medicine, The Catholic University of Korea, Seoul, Korea

Received: September 1, 2015

Revised : September 17, 2015

Accepted: September 18, 2015

\section{Correspondence to}

Chang Wook Kim, M.D.

Division of Hepatology, Department of Internal Medicine, College of Medicine, Uijeongbu St. Mary's Hospital, The Catholic University of Korea, 271 Cheonbo-ro, Uijeongbu 11765, Korea

Tel: +82-31-820-3997

Fax: +82-31-847-2719

E-mail: cwkim@catholic.ac.kr

\section{To the Editor,}

Although an association between pyogenic liver abscess and colon cancer has been suggested, liver abscess as a presenting sign of occult colorectal cancer has rarely been reported [1,2]. Destruction of the mucosal barrier by a colorectal neoplasm, followed by bacterial translocation into the portal venous system, might contribute to the formation of liver abscess [2]. We describe a case of recurrent pyogenic liver abscess at short intervals as the first manifestation of colorectal cancer in the absence of liver metastases. A healthy 66-yearold male visited the emergency room because of right upper abdominal pain associated with fever and chills over the past 5 days. He reported no history of cigarette smoking and alcohol consumption. Physical examination revealed tachycardia (pulse rate, 88 beats/ min), a body temperature of $38.7^{\circ} \mathrm{C}$, and tenderness of the right upper abdomen. Laboratory tests showed a white blood cell (WBC) count of $11,460 / \mu \mathrm{L}$, with $83.9 \%$ neutrophils, and a hemoglobin level of $14.4 \mathrm{~g} / \mathrm{dL}$. The blood chemistry profile was as follows: fasting glucose, $106 \mathrm{mg} / \mathrm{dL}$; total protein, $5.9 \mathrm{~g} / \mathrm{dL}$; albumin, $2.9 \mathrm{~g} / \mathrm{dL}$; total bilirubin, $0.9 \mathrm{mg} /$ $\mathrm{dL}$; aspartate aminotransferase, $107 \mathrm{U} / \mathrm{L}$; and alanine aminotransferase, $195 \mathrm{U} / \mathrm{L}$; alkaline phosphatase, $674 \mathrm{U} / \mathrm{L}$; gamma-glutamyl transferase, $321 \mathrm{U} / \mathrm{L}$. The
C-reactive protein level was $12.9 \mathrm{mg} / \mathrm{dL}$ (normal range < 0.3). Serologic tests for hepatitis B and C were negative. Serologic tests for tumor markers revealed normal levels of $\alpha$-fetoprotein (2.7 ng/ $\mathrm{mL}$ ), carbohydrate antigen 19-9 (9.1 U/ $\mathrm{mL}$ ), and carcinoembryonic antigen ( 0.7 $\mathrm{ng} / \mathrm{mL}$ ).

A computed tomography (CT) scan of the abdomen and pelvis demonstrated a multiseptated hypoechoic lesion in segment 6 of the liver, with a maximum diameter of $5.1 \mathrm{~cm}$ and poorly marginated margins (Fig. 1A). No other pathology was evident. A pyogenic liver abscess was suspected. Ultrasonography-guided percutaneous drainage and intravenous administration of a broad-spectrum antibiotic resulted in resolution of the fever and leukocytosis. Growth of Klebsiella pneumoniae was observed in pus cultures obtained from liver abscess aspirate and blood cultures. Cytology of liver abscess aspirate was negative for malignancy. The patient was discharged on oral antibiotic therapy after 4 weeks of hospitalization. A follow-up CT scan after 6 weeks of antibiotic therapy revealed regression of liver abscess (Fig. 1B).

Two months following his initial presentation, the patient revisited the emergency room suffering from fever and chills. His WBC count was 25,660/ $\mu \mathrm{L}$, with $80.4 \%$ neutrophils, and the 

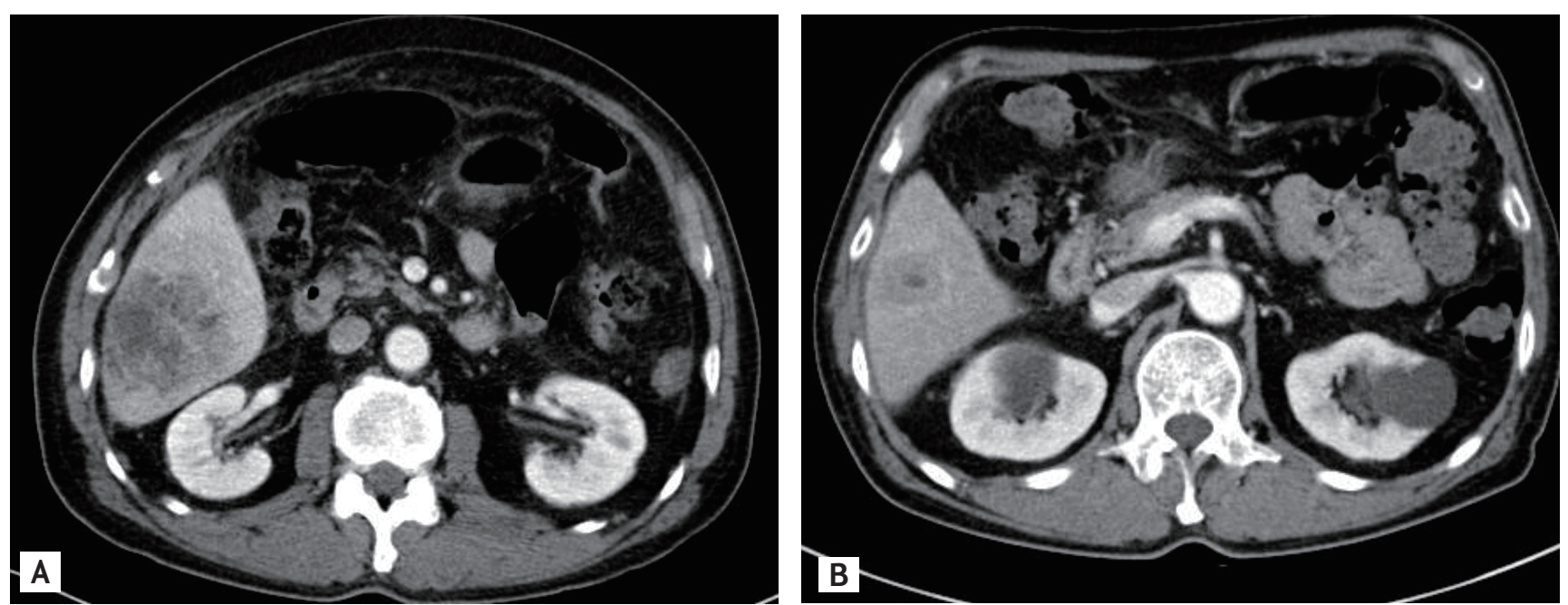

Figure 1. (A) Abdominal computed tomography (CT) scan shows a multiseptated abscess in segment 6 of the liver. (B) Follow-up CT scan 6 weeks after commencement of antibiotic treatment shows resolution of the lesion.
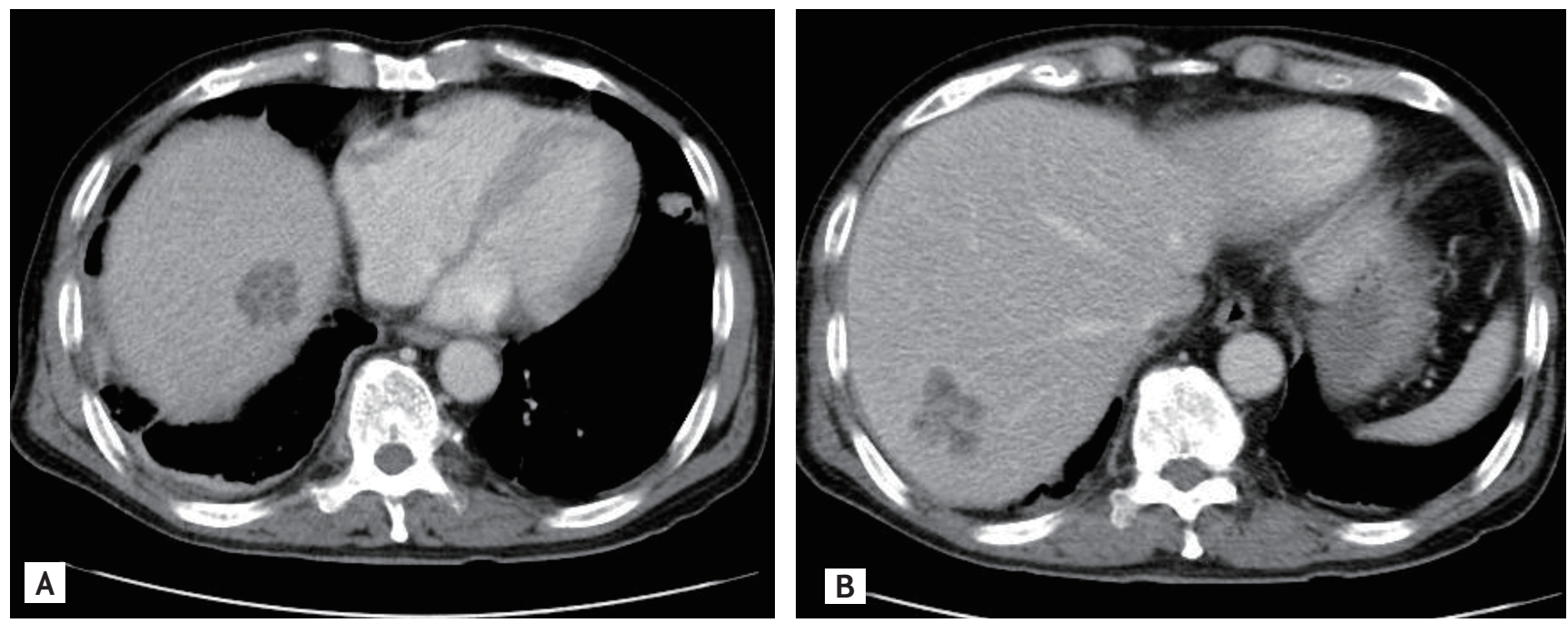

Figure 2. Abdominal computed tomography scan obtained at the second episode of liver abscess showed multiloculated abscesses in (A) segment 8 and (B) segment 7 of the liver.

C-reactive protein level was $14.9 \mathrm{mg} / \mathrm{dL}$. CT of the abdomen and pelvis revealed two newly developed hypodense lesions in segments $7 / 8$ of the liver, suggestive of liver abscess (Fig. 2). Moreover, eccentric thickening of the rectal wall, which was not seen in previous CT scans, was noted. Intravenous administration of antibiotics and percutaneous drainage were repeated. Blood and aspirated abscess cultures again showed growth of $K$. pneumoniae. Colonoscopy revealed an about $2.5 \mathrm{~cm}$ fungating mass located $4.5 \mathrm{~cm}$ from the anal verge (Fig. 3A). Histologic examination of the biopsy specimen disclosed a well-differentiated adenocarcinoma (Fig. 3B). Six weeks later, the patient underwent concurrent chemoradiotherapy. Sur- gical resection has been scheduled.

A systematic review of colorectal cancer-associated pyogenic liver abscess cases reported worldwide was published recently. In this report, K. pneumoniae was the predominant organism isolated from pyogenic liver abscess $(80 \%)$, and tumors were located mainly in the sigmoid colon and rectum (72.7\%) in Eastern Asian patients [3]. Mucosal defects in the neoplastic lesions are believed to facilitate entry of microorganisms into the portal circulation, with subsequent hematogenous spread to the liver [2].

Despite the recent evidence of an association between pyogenic liver abscess and colorectal cancer, the need for 

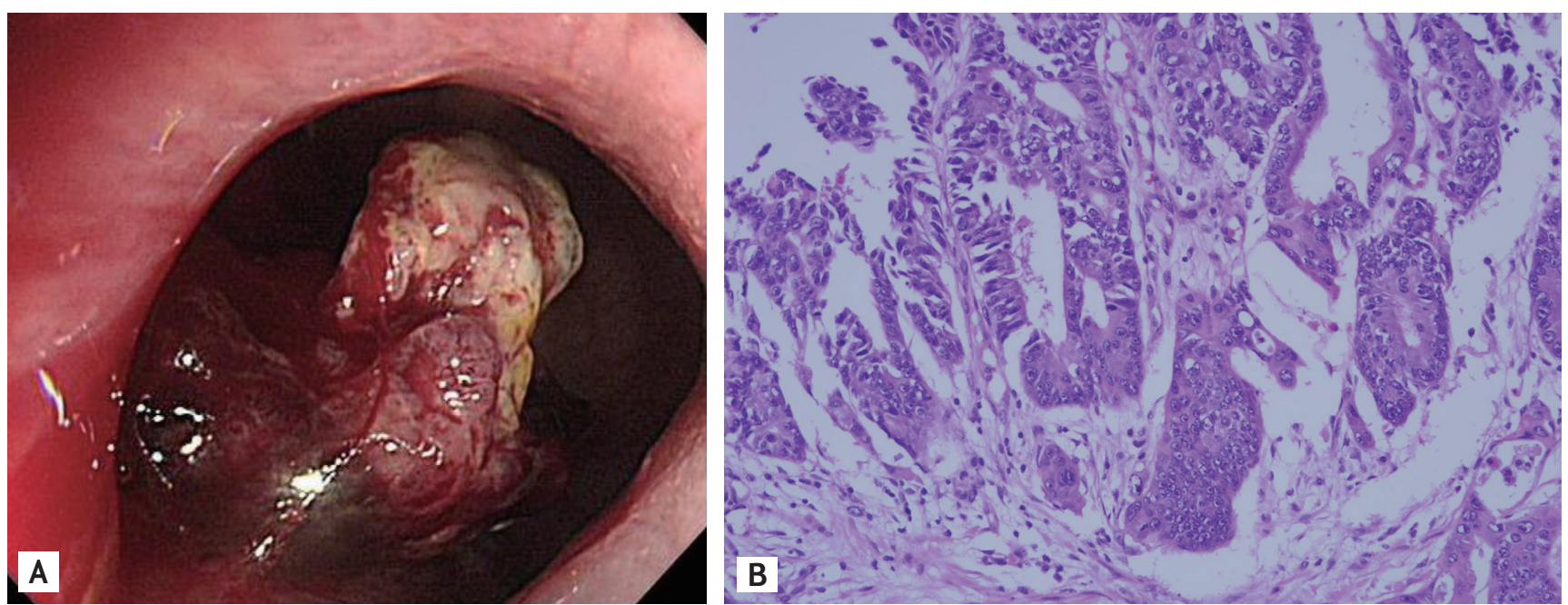

Figure 3. (A) Colonoscopy image shows an about $2.5 \mathrm{~cm}$ exophytic mass located $4.5 \mathrm{~cm}$ from the anal verge. (B) Microscopic examination of the biopsy specimen from the mass showed well-differentiated adenocarcinoma $(\mathrm{H} \& \mathrm{E}, \times 400)$.

colonoscopic investigation in patients with cryptogenic pyogenic liver abscess remains a matter of debate. In our case, colonoscopy was recommended based on accumulating evidence for a relationship between the pyogenic liver abscess and the underlying colorectal neoplasm, but the patient refused due to his general medical condition. Initially, CT of the abdomen and pelvis did not show any thickening of the colonic wall in this case. Hsu et al. [4] suggested that an abdominal CT scan inclusive of the pelvic area to evaluate liver abscess can assist in the diagnosis of colorectal cancer; however, the misdiagnosis of colorectal cancer in our case could be attributed to the collapsed bowel wall observed on the initial CT scan. Therefore, the possibility of colorectal neoplasm should be questioned in cases of cryptogenic pyogenic liver abscess, even if CT scans involving the pelvic area suggest no colonic pathology.

The patient did not complain of lower abdominal pain, changes in bowel habit, bloody stool, or tenesmus. Moreover, his carcinoembryonic antigen level was within normal limits. A recent review of pyogenic liver abscesses associated with colorectal cancers reported that only about $10 \%$ of the patients had colorectal cancer-associated symptoms. Moreover, colorectal cancer-related biomarkers were normal in most cases [3]. Therefore, the origin of pyogenic liver abscess should be identified in the absence of colorectal cancer-related symptoms or laboratory findings.

Recurrent liver abscess associated with colorectal neo- plasm has been reported only rarely. The time period between two subsequent episodes of pyogenic liver abscess was less than 1 year in most of these reports $[4,5]$. To the best of our knowledge, the case reported herein represents the earliest recurrence, at 2 months after the first manifestation of pyogenic liver abscess. As a result, colorectal cancer was diagnosed because of recurrent pyogenic liver abscess. However, a delay in the diagnosis of underlying colorectal cancer in our case led to recurrent liver abscess, which resulted in deferred treatment for colorectal cancer. Early investigation of the underlying cause of cryptogenic pyogenic liver abscess is warranted.

In conclusion, a pyogenic liver abscess can be the sole initial manifestation of colorectal cancer without associated metastasis. Increasing awareness of this association emphasizes the importance of skepticism regarding the presence of colorectal cancer in cases of otherwise cryptogenic pyogenic liver abscess. A thorough investigation, including colonoscopy, is necessary to identify the origin of an unexplained liver abscess. Timely diagnosis of common colonic pathologies associated with pyogenic liver abscess can improve the outcome of colorectal neoplasm. The cost-effectiveness of a complete evaluation of the gastrointestinal tract in patients with cryptogenic pyogenic liver abscess has not yet been elucidated.

Keywords: Colonic neoplasms; Colonoscopy; Liver abscess, pyogenic 


\section{Conflict of interest}

No potential conflict of interest relevant to this article was reported.

\section{REFERENCES}

1. Giuliani A, Caporale A, Demoro M, Scimo M, Galati F, Galati G. Silent colon carcinoma presenting as a hepatic abscess. Tumori 2007;93:616-618.

2. Teitz S, Guidetti-Sharon A, Manor H, Halevy A. Pyogenic liver abscess: warning indicator of silent colonic cancer. Report of a case and review of the literature. Dis
Colon Rectum 1995;38:1220-1223.

3. Qu K, Liu C, Wang ZX, et al. Pyogenic liver abscesses associated with nonmetastatic colorectal cancers: an increasing problem in Eastern Asia. World J Gastroenterol 2012;18:2948-2955.

4. Hsu WH, Yu FJ, Chuang CH, Chen CF, Lee CT, Lu CY. Occult colon cancer in a patient with diabetes and recurrent Klebsiella pneumoniae liver abscess. Kaohsiung J Med Sci 2009;25:98-103.

5. Lee JK, Kum J, Ghosh P. Nonmetastatic cancer of the colon associated with pyogenic liver abscess. Am J Gastroenterol 2008;103:798-799. 